\title{
LOAD BALANCING IN WDM NETWORK WITH SINGLE AND MULTIPLE LIGHTPATH ADDITIONS
}

\author{
M.Sumathi ${ }^{1}$, P.T.Vanathi ${ }^{2}$ \\ ${ }^{1}$ Department of ECE, Sona College of Technology, Salem, India - 643005 \\ E-mail: nnsuma@yahoo.com \\ ${ }^{2}$ Department of ECE, PSG College of Technology, Coimbatore, India - 641004 \\ E-mail: ptvani@yahoo.com
}

\begin{abstract}
Reconfiguration of a Wavelength Division Multiplexed (WDM) optical network is considered for varying traffic demands in the network. Reconfiguration is carried out with the aim of optimizing the objective function and minimizing the number of lightpath changes. This ensures the effective utilization of the network resources with minimum disruption to the network. In this paper, we present a new approach in which lightpaths with less traffic are considered for deletion during setup of a new lightpath. This increases the probability of setting up a new lightpath and decreases the value of the objective function; namely the congestion. Extensive simulation has been carried out for different traffic patterns. The congestion of the network and the number of lightpath changes has been studied. It is shown that deletion of lightpaths to free resources while setting up a new lightpath largely improves the congestion of the network. For slow varying traffic patterns, addition of one or two lightpaths minimized the congestion considerably. On the other hand, for rapid varying traffic patterns, minimizing load on one link might not considerably reduce congestion in the network. Therefore, multiple lightpath additions can enable decongesting multiple lightpaths in a single reconfiguration stage and balance the load in the network.
\end{abstract}

Keywords: Congestion, Lightpath, WDM, Dynamic Traffic, Mesh Network, Virtual Topology

\subsection{INTRODUCTION}

Optical fiber technology provides enormous bandwidth. Using WDM technology a number of wavelengths can be multiplexed and transmitted in a single fiber, thus enabling the utilization of the large bandwidth in the optical fiber. Optical amplifiers such as Erbium Doped Fiber Amplifiers (EDFA) can amplify a wide band of wavelengths. Hence, a single amplifier in the fiber can amplify all the wavelengths, eliminating the need for individual amplifiers for each wavelength. Each wavelength in a fiber can carry data of different formats and rates. Advancements in WDM technology further enable switching and routing in the optical domain. Different wavelengths in a fiber can be used to carry traffic which belongs to different source destination node pairs. Thus, lightpaths can be established between source destination node pairs. When a lightpath is established between node pairs, they become neighbours irrespective of their physical location. Data is optically switched at intermediate nodes over which the lightpath traverses and no conversion to electronic domain for processing occurs at these intermediate nodes. The nodes are equipped with Optical Cross Connects (OXC), which enable switching of the incoming wavelengths to outgoing wavelengths, and to source or sink wavelengths at the node. To set up a lightpath, it is necessary that a wavelength is available in the corresponding fiber links. In the absence of wavelength converters at the nodes, it is necessary that the same wavelength is available in the fiber links in order to establish a lightpath.

The set of all lightpaths in a network forms the virtual topology. Virtual topology is the topology seen by the higher layers. The advantage of virtual topology is that it can be reconfigured whenever necessary. The design of a virtual topology for the given physical topology requires virtual topology selection, lightpath route selection, lightpath wavelength selection and routing of traffic over the virtual topology [1]. We have considered a combined approach of solving the above sub-problems. Shortest path algorithm is used for lightpath route selection, and for routing of packets over the virtual topology. Among the objectives that can be considered for virtual topology design are including minimal congestion, delay and average weighted number of hops [1]. An extensive survey of virtual topology design is carried out by Dutta and Rouskas [2], where problem formulation for virtual topology design is elaborated and various linear programming and heuristic methods are discussed. Linear programming method becomes intractable for large networks; hence, heuristics that give optimal solutions are useful. The study of lightpath establishment for static and dynamic environment is found in Dutta and Sengupta [3]. Research on virtual topology design for different topologies has been carried out considering one or more objective functions. For example, virtual topology designed with minimal packet delay, minimal congestion, optical resource cost reduction 
as well as minimal number of rejected connections can be found in [4][5][6][7][8] and [9]. On another perspective, combined problem of physical and virtual topology design using genetic algorithm is proposed in [10].

Developments in networking are already moving towards designing an intelligent network which can dynamically adapt to real time environments. For this reason, it is necessary that an optical network is capable to be reconfigured which may be caused due to network component failure or dynamic traffic. When a node (or a link) fails, or when the traffic conditions in the network vary, the virtual topology needs to be reconfigured in order to optimize the network performance. It is necessary that the reconfiguration process cause minimal disruption while optimizing the performance. The same objective as that of virtual topology design can be used for reconfiguration process. Reconfiguration using linear programming method to minimize the objective function and the number of lightpath changes for a mesh network is proposed in [11]. Linear programming methods and heuristic approaches can be used for reconfiguration. Linear programming is found to be computationally intractable. Therefore, heuristic approaches are preferred. A two-stage approach to virtual topology reconfiguration is proposed in [12], where average weighted hop count is considered as the objective function. The reconfiguration stage determines the lightpath changes required and the optimization stage that aims at minimizing the deviation from the objective function value. An adaptive reconfiguration mechanism which does not require prior knowledge of the future traffic pattern is discussed in [13]. We consider addition (or deletion) of a lightpath for a slow varying traffic pattern. When the load of more than one lightpath is outside the watermark region, a variation to the basic approach is proposed. In this case, multiple lightpath additions or deletions are allowed. Lightpath addition (or deletion) is triggered when the maximum load in a link exceeds the higher watermark level (WH) (or below the lower watermark level (WL)). We consider both cases (either addition or deletion) of one or more lightpaths during each stage of adaptation. A lightpath cannot be added if resources such as transmitter at the source node, receiver at the destination node, or wavelengths along shortest path on the physical link are not free. Deletion of a lightpath is considered only when a lightpath cannot be added. We discuss a new approach in which lightpaths with less traffic are considered for deletion during the setup of a new lightpath. Our proposal offers minimal congestion in the network with few additional lightpath changes.

The rest of the paper is organized as follows. In Section 2, we discuss the problem specification and the parameters used. In Section 3, we discuss the heuristic algorithm. In Section 4, we discuss the traffic model and simulation results. In Section 5, we present our conclusion.

\subsection{PROBLEM SPECIFICATION}

A centralized heuristic approach to the reconfiguration problem is considered. The WDM mesh network with $\mathrm{N}$ nodes has bi-directional optical links connecting the nodes. W wavelengths are available in each direction. $\mathrm{TR}_{\mathrm{i}}$ indicates the number of transceivers in each node. The physical topology connectivity matrix, PT gives the physical connection between the nodes. The virtual topology designed over the physical topology is connected by unidirectional lightpaths. The virtual topology connectivity matrix, VT gives the lightpath connection between the nodes. The connectivity between all nodes in the virtual topology is assumed. Wavelength continuity constraint is relaxed, and it is assumed that all nodes are equipped with the wavelength converters. Hence, while establishing a lightpath, it is not necessary that the same wavelength to be available on all links. It is assumed that at least one lightpath exists between any node pair. T denotes the traffic matrix, where $t_{\mathrm{s}, \mathrm{d}}$ denotes the traffic from source, $s$ to destination, $\boldsymbol{d}$. $\mathrm{L}_{\mathrm{ch}}$ denotes the number of lightpath changes (number of lightpaths added and deleted). WH indicates the upper threshold of traffic for triggering the reconfiguration process, and $\mathrm{L}_{\text {range }}$ denotes the range of lightpath changes. The objective function congestion is defined as the maximum traffic flow in a lightpath due to all source destination node pairs and this is given by [7].

$$
\lambda_{\max }=\max _{i, j} \lambda_{i, j}
$$

The total traffic on the virtual link from node $\mathbf{i}$ to $\mathbf{j}$ is given by

$$
\lambda_{i j}=\sum_{s, d} \lambda_{i j}^{s d}
$$

where $\lambda_{i j}^{s d}$ gives the traffic from node $s$ to $\boldsymbol{d}$ which employs the virtual link $\mathbf{i}$ to $\mathbf{j}$.

Objective of the algorithm is to minimize congestion, while maintaining the number of lightpath changes within a specific range. In order to accommodate new lightpaths, existing lightpaths with less traffic may be deleted. The virtual topology is designed for the new traffic matrix considering physical topology, existing virtual topology and 
resource constraints. Shortest path algorithm is used to determine the path for a lightpath over the physical topology, and for routing of traffic over the virtual topology.

\subsection{HEURISTIC ALGORITHM}

In our proposed method, the optimal virtual topology that has the minimum value of congestion and number of lightpath changes within specific range is obtained for the given traffic matrix. First, congested lightpath in the network is determined. The source destination node pairs that use this lightpath are determined and a new lightpath is established (between the node pair). New establishment of lightpath enables to divert the traffic from the congested link. The heuristic algorithm considers the congested lightpath in the network. If congestion is greater than WH, all source destination node pairs that use the lightpath $\mathbf{i}, \mathbf{j}$ are determined. Starting from the source destination node pair with maximum traffic, an attempt is made to establish a new lightpath in order to minimize the congestion and to maintain the number of lightpath changes. If resources are not available to establish the lightpath, then existing lightpaths (which use the resources) along the shortest path are considered for deletion. Detail of the heuristic algorithm is as follows:

1. begin

2. For the new traffic matrix determine the congestion in the network and the link $\mathbf{i}, \mathbf{j}$ (which is most congested).

3. if $\left(\lambda_{\max }>\mathrm{WH}\right)$

4. Determine all source destination node pairs that are using the link $\mathbf{i}, \mathbf{j}$ during the multihopping

5. Sort the node pairs $s_{m}, d_{n}$ in descending order of traffic between them, and

6. for each source destination node pair $\left(\mathrm{s}_{\mathrm{m}}, \mathrm{d}_{\mathrm{n}}\right)$

7. Determine the shortest path in the physical topology between $s_{m}, d_{n}$.

8. if resources are not free

9. Determine the combination of lightpaths to be released (to free the resources)

10. Obtain the sets of these lightpaths to be released

11. for each lightpath set

12. Delete lightpaths to free resources

13. Establish lightpath between nodes $s_{m}$ and $d_{n}$

14. if new virtual topology is connected and congestion $<$ previous congestion value and $\mathrm{L}_{\mathrm{ch}}<\mathrm{L}_{\text {range }}$

15. Accept this as new reconfigured topology

16. end if

17. end for

18. end if

19. end for

20. end if

21. end

The single lightpath addition (SLPA) algorithm as described considers one congested lightpath $\mathbf{i}, \mathbf{j}$. Setting up a new lightpath diverts the traffic over this lightpath. The same concept can be carried out considering $\boldsymbol{k}$ congested lightpaths, called as multiple lightpath addition (MLPA) algorithm. For a network with rapid change in traffic pattern, a single reconfiguration stage can balance the overall network load. The work in [13] proposed an approach where a single lightpath is either added or deleted during each reconfiguration stage via single lightpath addition or deletion (S_ADN) algorithm and multiple lightpaths via multiple lightpath addition or deletion (M_ADN) algorithm. In comparison, via SLPA and MLPA algorithms our method consider deletion of existing lightpaths to free resources for establishment of new lightpaths. 


\subsection{SIMULATION RESULTS}

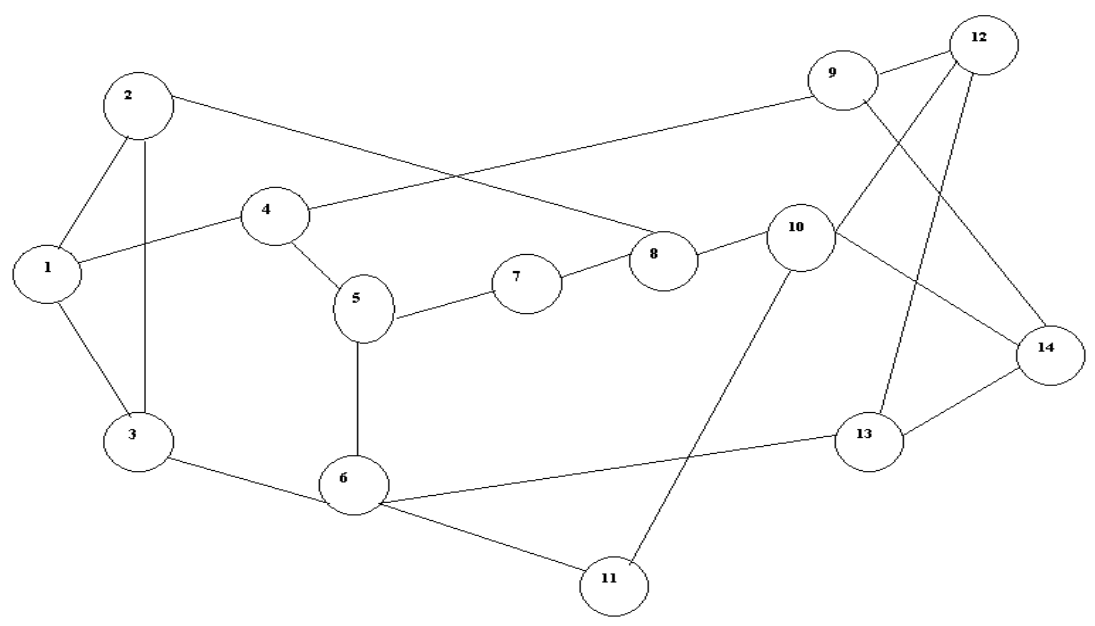

Fig.1 NSFNET topology

We considered fourteen nodes NSFNET T1 network with 21 bi-directional links (Fig.1) as the sample network. Each node is assumed to have eight transceivers, $\mathrm{TR}=8$ (i.e. eight transmitters and eight receivers) and a fiber link supporting eight wavelengths, $\mathrm{W}=8$. The traffic matrix is randomly generated with uniform distribution [12]. A certain fraction $\mathbf{F}$ of the traffic is uniformly distributed over the range $[0,(\mathrm{C} / \mathrm{a})]$ and the remaining over the range $[0$, $\left(C^{*} \gamma / \mathbf{a}\right)$ ], where $\mathbf{C}$ represents the lightpath capacity, $\boldsymbol{a}$ is an integer and $\gamma$ is the average traffic intensities between node pairs with high and low traffic values. The traffic is normalized with respect to the capacity of a lightpath. The values of $\mathrm{a}=20, \gamma=10$ and $\mathrm{F}=0.70$ are used for the simulation purpose. Two types of traffic patterns have been generated for the study. The first traffic pattern considers changes in traffic percentage and the other changes in time period.

Changes in traffic percentage:

The traffic change is obtained by swapping a certain fraction $\mathrm{x} \%$ of the non-diagonal entries in the traffic matrix. The percentage of variation of $\mathbf{x}$ is varied for each instance between 10 and 80 .

Dynamic traffic for different time periods:

The traffic sequence is obtained for a certain fraction of traffic change. Continuous change in traffic is obtained by linear interpolation between two consecutive values. Two dynamic traffic patterns for $20 \%$ and $40 \%$ traffic change have been obtained.

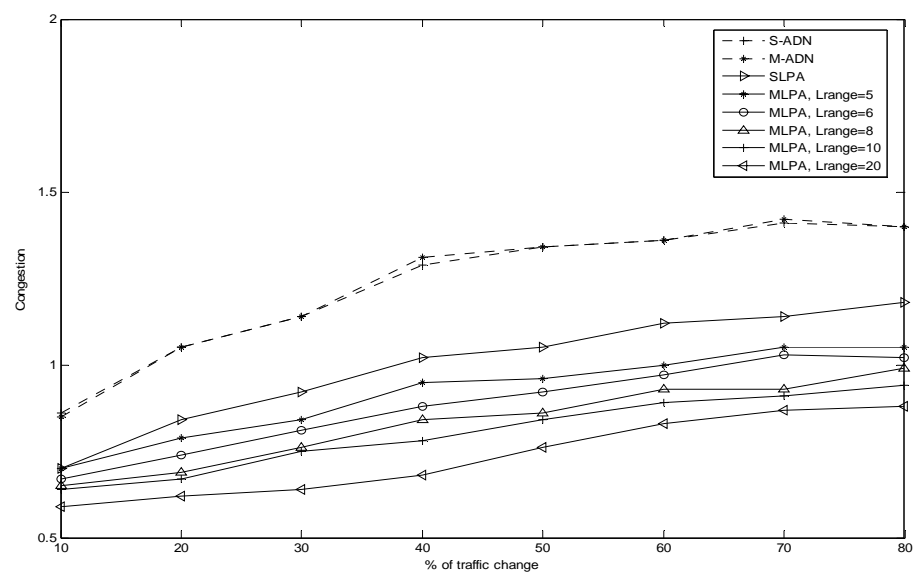

Fig. 2 Congestion for different values of $\mathrm{L}_{\text {range }}$ with $\mathrm{WH}=40$ and $\mathrm{k}=10$ for MLPA 


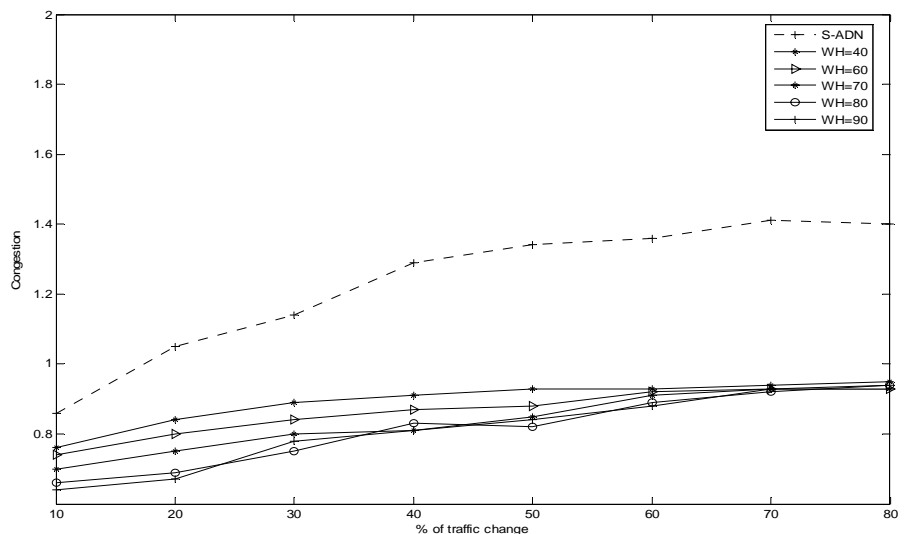

Fig. 3 Congestion for different values of WH for MLPA with $\mathrm{L}_{\text {range }}=10$ and $k=10$

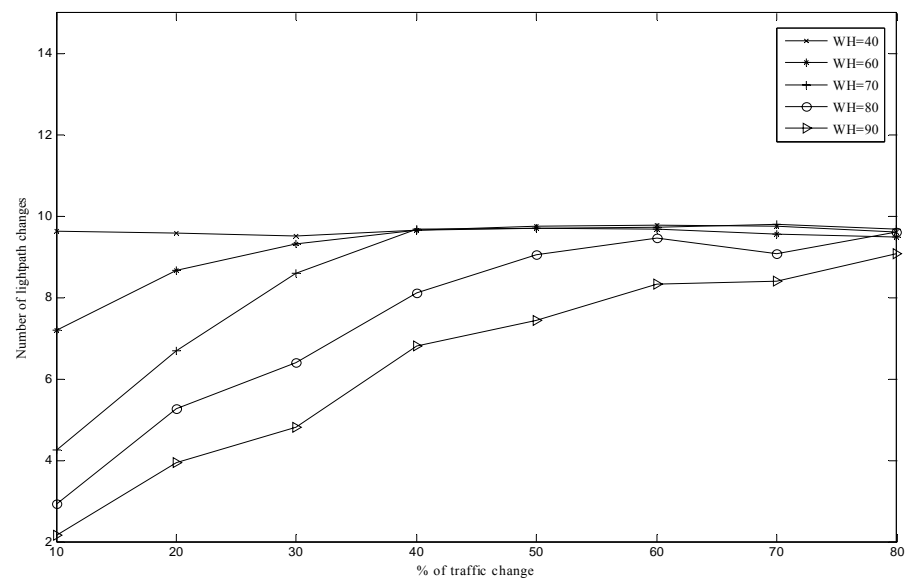

Fig. $4 \mathrm{~L}_{\mathrm{ch}}$ for different values of $\mathrm{WH}$ for MLPA with $\mathrm{L}_{\text {range }}=10$ and $\mathrm{k}=10$

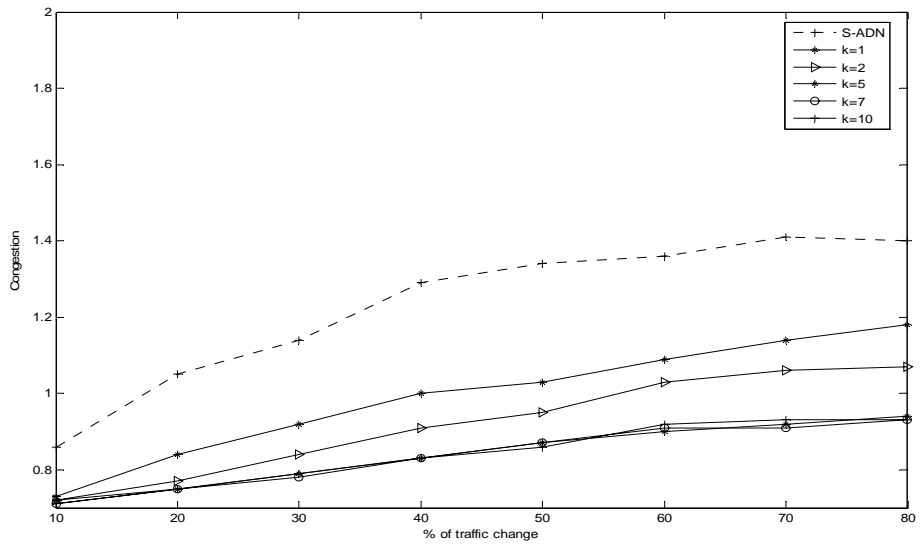

Fig.5 Congestion for different values of $\mathrm{k}$ with $\mathrm{WH}=80$ and $\mathrm{L}_{\text {range }}=10$ 
Fig. 2 to Fig. 5 shows the results when changes in traffic percentage occurred. In Fig.2, the congestion of the network is plotted. The SLPA algorithm results in a lower value of congestion compared to the S_ADN algorithm which considers either addition or deletion of a lightpath during each stage. The S_ADN algorithm establishes a lightpath only if resources are free; whereas the SLPA algorithm deletes some of the existing lightpaths to free resources (if resources are not available) in order to minimize the congestion in the network. Thus, the probability of establishing a new lightpath increases. The M_ADN approach did not show much improvement in minimizing the congestion as compared to the S_ADN algorithm. This is due to unavailability of resources to establish a new lightpath. On the other hand, the congestion for MLPA algorithm is lesser than that of SLPA algorithm. Further, we note that as the number of allowed lightpath changes $\left(\mathrm{L}_{\text {range }}\right)$ increases, the value of congestion decreases. The results for congestion for different values of WH are shown in Fig. 3 with value $\mathrm{L}_{\text {range }}=10$ and $\mathrm{k}=10$ respectively.

For lower values of percentage of traffic change, congestion of network depends on the threshold value, WH. However, for larger values congestion remained the same independent of WH value. This is due to the fact that for higher percentage of traffic change, congestion is larger and reconfiguration is triggered for all WH values. Fig.4 shows that when the percentage of traffic change is small, the number of lightpath changes increases for smaller values of WH. As the percentage of traffic change increases, the difference in number of lightpath changes (for different values of $\mathrm{WH}$ ) decreases.

The congestion in the network for different approaches is plotted as in Fig.5. As we have discussed, S_ADN approach results in higher value of congestion (either whenever addition or deletion of a lightpath occurs). Compared to SLPA, MLPA allows further decrease in congestion since the probability of establishing new lightpaths is higher. We observed that increasing the value of $\mathrm{k}$ did not improve (or decrease) the network congestion.

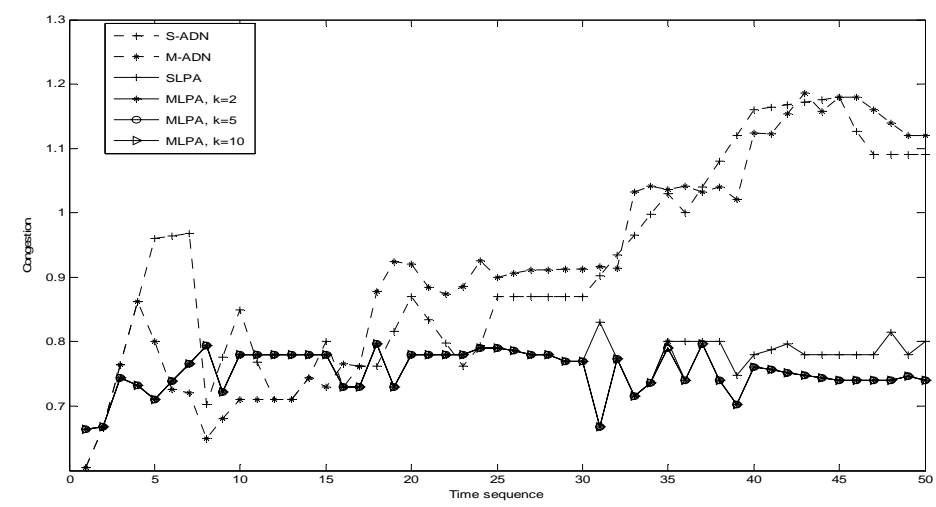

Fig. 6 Congestion for dynamic continuous traffic with $\mathrm{WH}=80$ and $\mathrm{L}_{\text {range }}=10$ and traffic change $=20 \%$

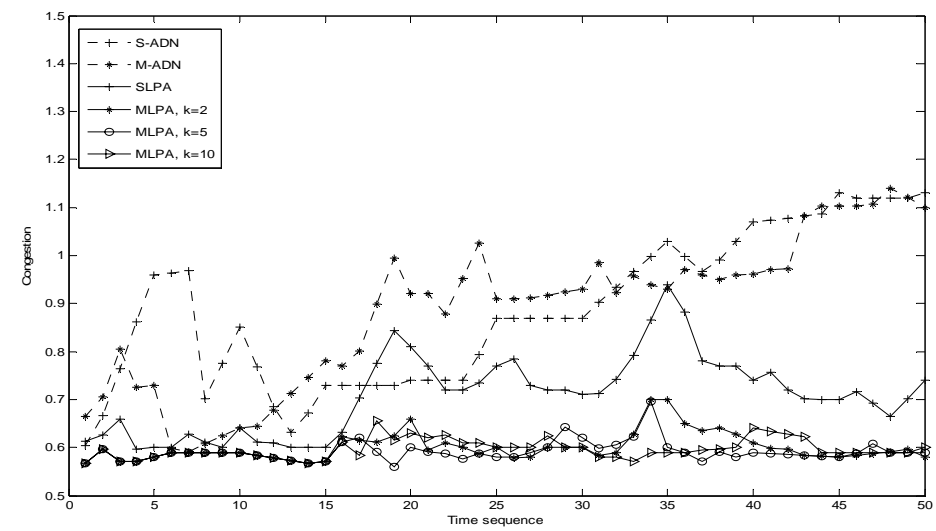

Fig.7 Congestion for dynamic continuous traffic with $\mathrm{WH}=60$ and $\mathrm{L}_{\text {range }}=10$ and traffic change $=20 \%$ 


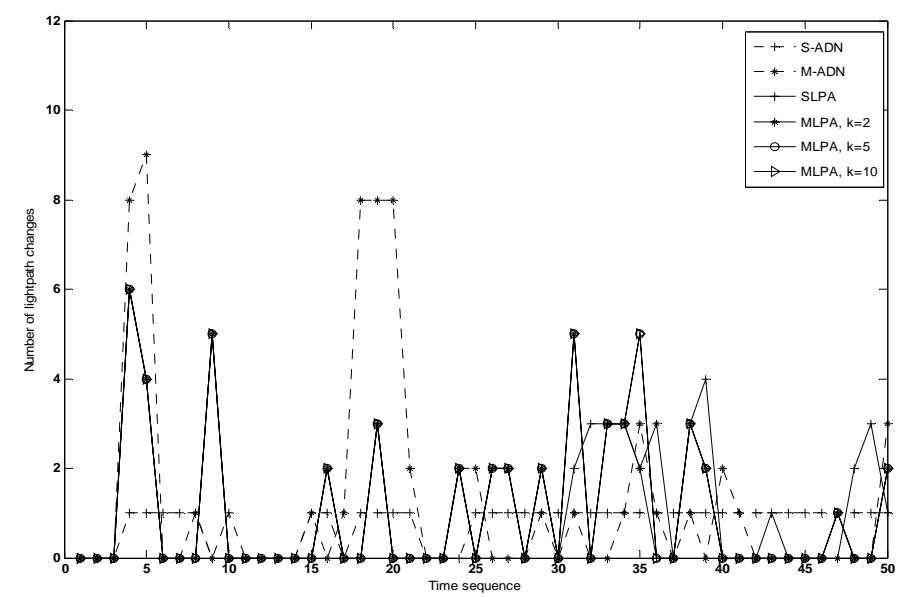

Fig.8 $\mathrm{L}_{\mathrm{ch}}$ for dynamic continuous traffic with $\mathrm{WH}=80$ and $\mathrm{L}_{\text {range }}=10$ and traffic change $=20 \%$

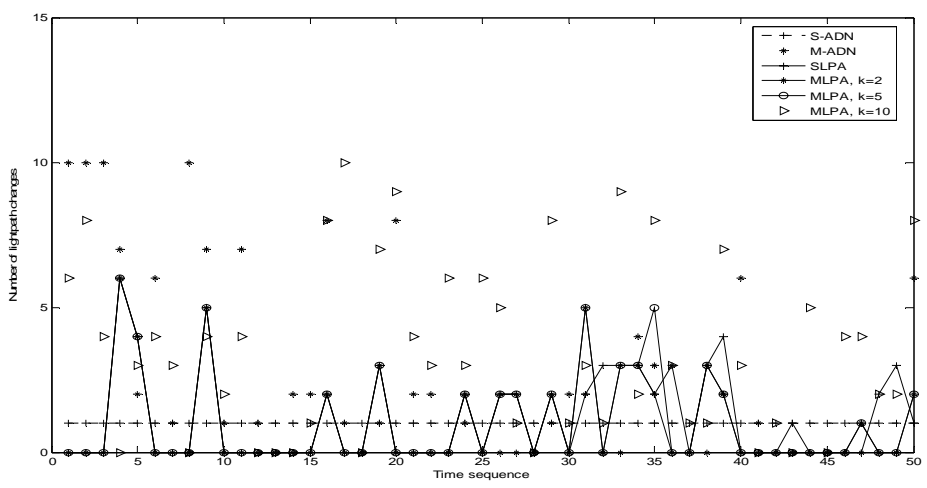

Fig.9 $\mathrm{L}_{\mathrm{ch}}$ for dynamic continuous traffic with $\mathrm{WH}=60$ and $\mathrm{L}_{\text {range }}=10$ and traffic change $=20 \%$

Simulation results for time varying continuous traffic are given in Fig.6 to Fig.9. As illustrated, Fig.6 and Fig.7 show the congestion in the network with values $\mathrm{WH}=80$ and $\mathrm{WH}=60, \mathrm{~L}_{\text {range }}=10$ and traffic changes $=20 \%$ respectively. Fig. 8 and Fig.9 show the corresponding values of lightpath change. As we noted earlier, SLPA and MLPA algorithms result in lesser congestion. When multiple lightpaths are considered during reconfiguration, the congestion in the network reduces and is maintained almost at the threshold value. For lower values of $\mathrm{WH}$, the number of lightpath changes is larger.

\subsection{CONCLUSION}

The traffic generated by the higher layers is dynamic. For that reason, reconfiguration of virtual topology is done to maximize utilization of resources. Reconfiguration should consider the number of lightpath changes to minimize the disruption of the network. A centralized approach to reconfiguration has been considered with congestion as the objective function. Reconfiguration process considers addition of new lightpaths to divert traffic from congested lightpaths. In this work, deletion of existing lightpaths carrying less traffic is considered to accommodate new lightpaths. Deletion of lightpath also affects the congestion in the network.

Simulation results proved that the addition of lightpaths can considerably improve (or decrease) the congestion of the network. However, increasing the number beyond a certain value did not really improve the network performance. The value of WH determines the congestion value of the network and also the number of lightpath changes. 
A distributed approach to the same problem of reconfiguration can be considered. In this work, congestion has been considered as the objective function, in addition to lightpath changes. Further work could include considering two or more objective functions simultaneously, so that other objective functions do not deviate from their optimal values. Future work will be extended to include multiple lightpaths between the node pairs. Altogether, the wavelength continuity constraint may also be considered.

\section{REFERENCES}

[1] C.SivaRamMurthy and M.Gurusamy, WDM Optical Networks: Concepts, design and algorithms, PHI, 2002.

[2] R.Dutta and G.N.Rouskas, "A survey of VT design algorithms for wavelength routed optical networks", TR-9906, May 12, 1999.

[3] Raja Dutta and Indranil Sengupta, "Static and Dynamic Connection Establishment in WDM Optical Networks: A Review”, IETE Journal of Research, May/June 2005, pp.209 - 221.

[4] Amrinder S.Arora, Suresh Subramaniam, Hyeong-Ah Choi, "Logical Topology Design for Linear and Ring Optical Networks”, IEEE Journal on Selected Areas in Communications, vol. 20(1), January 2002, pp.62-74.

[5] B.Mukherjee, D.Banerjee, S.Ramamurthy, A.Mukherjee, "Some principles for designing a wide area WDM optical network”, IEEE/ACM Transactions on Networking, vol. 4(5), October 1996, pp.684-696.

[6] Rajesh M. Krishnaswamy, Kumar N. Sivarajan, "Design of Logical Topologies: A Linear Formulation for Wavelength-Routed Optical Networks with No Wavelength Changers", IEEE/ACM Transactions on Networking, vol. 9(2), April 2001, pp.186-198.

[7] R.Ramaswami, K.N.Sivarajan, "Design of logical topologies for wavelength routed optical networks", IEEE Selected Areas in Communication, vol. 4(5), June 1996, pp.840-851.

[8] M.Sumathi and P.T.Vanathi, "Minimizing Congestion in Optical WDM Networks for Dynamic Traffic", in Proc. of IEEE INDICON 2005, IIT, Chennai, India, Dec. 2005, pp. 101-104.

[9] Stefano Bregni, Ugo Janigro, Achille Pattavina, "Optimal allocation of limited optical layer resources in WDM networks under static traffic demand”, Photonic Network Communications, vol. 5(1), January 2003, pp.248252.

[10] Y.Xin, G.N.Rouskas, H.G.Perros, "On the physical and logical topology design of large scale optical networks", Journal of lightwave technology, vol. 21(4), April 2003, pp.904-915.

[11]D.Banerjee, B,Mukherjee, "Wavelength-routed optical networks: Linear formulation, Resource Budgeting tradeoffs and a reconfiguration study", IEEE/ACM Transactions on Networking, vol. 8(5), October 2000, pp.598-607.

[12] N.Sreenath, B.H.Gurucharan, G.Mohan, C.Siva Ram Murthy, "A two-stage approach for virtual topology reconfiguration of WDM optical networks", Optical Networks Magazine, May/June 2001, pp.58-71.

[13] A.Gencata, B.Mukherjee, "Virtual topology adaptation for WDM mesh networks under dynamic traffic", IEEE/ACM Transactions on Networking, vol. 11(2), April 2003, pp.236-247.

[14] Anuradha Narula_Tam, Eytan Modiano, "Dynamic load balancing in WDM packet networks with and without wavelength constraint”, IEEE Journal on Selected Areas in Communication, vol. 18(10), October 2000, pp. 1972-1980.

[15] R.Ramaswami and K.N.Sivarajan, Optical Networks: A practical perspective, Morgan Kaufmann Pub., 1998. 


\section{BIOGRAPHY}

M. Sumathi received the B.E degree in Electronics and Communication Engineering from PSG College of Technology, Coimbatore, India in 1992 and the M.E degree in Optical Communication from College of Engineering, Chennai, India in 2004. Currently she is pursuing her doctoral degree under Anna University, Chennai, India. She was a recipient of the post graduate scholarship, sponsored by the government of India, from 2002 to 2004. From 1992 to 1993 she was a Systems Analyst at Sonata Software Limited, Bangalore, India. She joined Sona College of Technology, Salem as lecturer in 1999, where she is currently Assistant Professor in the department of Electronics and Communication Engineering. Her research interest includes WDM networks, optical communication, computer networks and wireless communication. She is a member of IEEE, OSA and ISTE.

P. T. Vanathi received the B.E degree in Electronics and Communication engineering from PSG College of Technology, Coimbatore, India in 1985 and the M.E degree in Applied Electronics from the same institution in 1991. She has completed her Ph.D in signal processing. She is a Professor in the department of Electronics and Communication engineering, PSG College of Technology, Coimbatore. She has 19 years of teaching experience. Her research interest includes speech recognition, network security and signal processing. She is a member of ISTE and IETE. 